\title{
Inferior Vena Cava Collapsibility Index and Central Venous Pressure for Fluid Assessment in the Critically III Patient
}

\author{
Afia Arshed Dodhy \\ Department of Anaesthesia \& ICU, Lahore General Hospital, PGMI/LGH/AMC, Lahore, Pakistan
}

\begin{abstract}
Objective: To determine the correlation of sonographic evaluation of inferior vena cava diameter and its collapsibility index with central venous pressure in both spontaneously breathing and mechanically ventilated patients in surgical ICU.

Study Design: Cross-sectional study.

Place and Duration of Study: Surgical ICU, Lahore General Hospital from November 2020 to May 2021.

Methodology: All patients above 18 years of age, who had central venous catheter placed, were included in the study. Patients with raised abdominal pressure, pregnancy, morbid obesity, heart diseases, and those unable to lie in supine position, were excluded. For inferior vena cava (IVC) measurement, IVC was visualised using curvilinear probe of ultrasound machine. Minimum and maximum diameters of inferior vena cava were calculated in every respiratory phase. IVC collapsibility index was expressed in percentage. Central venous pressure (CVP) was recorded soon after IVC measurement, using manometer.

Results: Total number of patients was 126. A significant correlation was seen between IVC measurements (inferior vena cava diameters and the collapsibility index) and CVP, $(p<0.001)$, but the regression coefficients were less in patients who were mechanically ventilated ( $r=0.779$ for IVC maximum diameter and -0.725 for collapsibility index) than the patients who were breathing spontaneously ( $r=0.850$ for IVC maximum diameter and -0.899 for collapsibility index)

Conclusion: Evaluation of IVC diameter and its collapsibility index is an easy and non-invasive method to evaluate intravascular volume status of critically ill patients. Its use is more helpful in patients who are spontaneously breathing than those who are mechanically ventilated.
\end{abstract}

Key Words: Central venous pressure, Fluid status, Inferior vena cava diameter.

How to cite this article: Dodhy AA. Inferior Vena Cava Collapsibility Index and Central Venous Pressure for Fluid Assessment in the Critically III Patient. J Coll Physicians Surg Pak 2021; 31(11):1273-1277.

\section{INTRODUCTION}

Determination of intravascular plasma volume and fluid management, in critically sick patients of intensive care units, is of utmost importance for targeted management. ${ }^{1}$ Numerous methods are used to evaluate the intravascular fluid status for effective management. Commonly, measurement of central venous pressure through central venous catheter (CVC) is used for this objective but routine and extensive use of this invasive technique is restricted due to many complications related to it. ${ }^{2} \mathrm{Few}$ common CVC related complications are arterial puncture, pneumothorax, arrhythmias and catheter-associated infections. $^{3}$

Correspondence to: Dr. Afia Arshed Dodhy, Department of Anaesthesia \& ICU, Lahore General Hospital, PGMI/LGH/AMC, Lahore, Pakistan

E-mail: afiashujaat@yahoo.com

Received: August 03, 2021; Revised: September 09, 2021;

Accepted: October 04, 2021

DOI: https://doi.org/10.29271/jcpsp.2021.11.1273
As standard of care change to minimal and non-invasive monitoring techniques; sonographic evaluation of the IVC signifies an efficient and non-invasive technique for assessing fluid status. ${ }^{2}$ It is seen that calculation of IVC diameters and its collapsibility index, i.e. IVC-Cl correlate with intravascular fluid status and respond to clinical interventions. ${ }^{4}$

The IVC is a very compliant major vein, which alters its diameter with changing fluid status of the body. ${ }^{5}$ It expands and contracts with each breath. During inspiration, negative pressure is created, which increases venous return to heart, thereby transitory collapsing IVC. ${ }^{5}$ Conversely, during exhalation, venous return is decreased and diameter of the IVC resumes to its baseline. ${ }^{5}$ In decreased intravascular fluid status, the percentage collapse of this vein is correspondingly greaterthan in increased intravascularfluid status. ${ }^{5}$

Ultrasound is freely available at bedside in critical care setups. It is non-invasive, economical and safe. Moreover, it can provide rapid assessment of intravascular volume status with littleexpertise. ${ }^{6}$ 
The present study was carried out to see the correlation of sonographic evaluation of IVC diameter and its collapsibility index with central venous pressure and thus to practice, assessment of IVC, as a guide for intravascular fluid status. Furthermore, to find, whether the relationship is same or different in both mechanically ventilated and spontaneously breathing patients admitted in critical care unit.

\section{METHODOLOGY}

After taking ethical approval from Research Review Committee of the Institution, (Research No.00-172-20), this cross-sectional study was carried out in Surgical ICU, Lahore General Hospital from November 2020 to May 2021. Written informed consent was taken from patients, who were on spontaneous breathing; and from legal guardians of patients, who were on mechanical ventilation, before participation and were notified regarding the research study.

All patients above 18 years of age, who had central venous catheter placed, were included in the study. Patients with raised abdominal pressure, pregnancy, morbid obesity, heart diseases, unable to lie in supine position and where ultrasound was not possible due to wound dressings, were excluded from the study. Power analysis and sample size (PASS 2018) was used to calculate the sample size. A sample size of 126 achieves $90.132 \%$ power to detect an odds ratio of 3.286, using a two-sided McNemar test with a significancelevel of 0.050 .

Patients in whom central venous catheter was passed for medical indications were evaluated for study. Their demographic data was recorded. For IVC measurement, Mindray ultrasound machine was used in all patients. All examinations were done in a blind fashion by the same intensivist; and were not appraised by the CVP value and patient's hemodynamics. Curvilinear probe was placed at subxiphoid region in the supine position of the patient. IVC was visualised in cross-sectional view and then rotated to obtain its long axis view. Then, M mode was used and minimum and maximum anterio-posterior internal diameters of IVC were measured at $3 \mathrm{~cm}$ inferior from right atrium border in each respiratory cycle. IVC-Cl was expressed in percentage by calculating maximum diameter in $\mathrm{mm}$ on expiration, minus minimum diameter in mm on inspiration, and dividing by maximum diameter in mmon expiration.

CVP was recorded soon after IVC measurement. In the supine position, CVP was measured manually, using manometer. Reference point was taken at the intersection point of mid- axillary line with the fourth costal cartilage and measurement was recorded in $\mathrm{cm}$ of $\mathrm{H}_{2} \mathrm{O}$.

For analysis of data, SPSS version 23 was used. Total number of patients enrolled in the study was 126 . Out of these, 67 patients were on mechanical ventilation and 59 patients were on spontaneousventilation.

\section{RESULTS}

Among 126 patients, 54 patients (42.9\%) were females and 72 patients $(57.1 \%)$ were males. Average age of the patients was 42 years with minimum of 22 years and maximum of 58 years. Distribution of patients by diagnosis at time of admission in ICU is shown in Tablel.

\begin{tabular}{l} 
Table I: Patient characteristics. \\
\begin{tabular}{|l|l|l|}
\hline Characteristics & Number (n) & Percentage (\%) \\
\hline Total patients & 126 & \\
\hline On mechanical ventilation & 67 & 53.1 \\
\hline On spontaneous ventilation & 59 & 46.9 \\
\hline Age (mean \pm SD) & $42 \pm 10.4$ & \\
\hline Gender & 54 & 42.9 \\
\hline Female & 72 & 57.1 \\
\hline Male & \multicolumn{2}{|l|}{} \\
\hline Diagnosis (at admission) & 26 & 20.6 \\
\hline Gastrointestinal surgery & 16 & 12.7 \\
\hline Caesarean section & 22 & 17.5 \\
\hline Trauma (post-surgery) & 15 & 11.9 \\
\hline Genitourinary surgery & 16 & 12.7 \\
\hline Hysterectomy / gynaecology surgery & 10 & 7.9 \\
\hline Orthopaedic surgery & 11 & 8.7 \\
\hline Trauma (non-operative) & 10 & 7.9 \\
\hline Other &
\end{tabular} \\
\hline
\end{tabular}

CVP values with their corresponding IVC maximum diameter and collapsibility index in spontaneously and mechanically ventilated patients are presented in Tablell.

To find the co-relation between CVP and IVC measurements (IVC diameters and the collapsibility index), Pearson test was applied. However; a significant correlation statistically, both in spontaneously breathing and mechanically ventilated patients, was seen $(p<0.001)$, but the regression coefficients were less in patients who were mechanically ventilated $(r=0.779$ for IVC maximum diameter and -0.725 for collapsibility index) than the patients who were breathing spontaneously ( $r=0.850$ for IVC maximum diameter and -0.899 for collapsibility index). The results are tabulated in Table III.

\section{DISCUSSION}

In the present study, it is seen that ultrasonic evaluation of IVC measurements (diameter of IVC and collapsibility index) is significantly correlated with the CVP measurement in both mechanically ventilated as well as patients breathing spontaneously. However, this correlation was much stronger in spontaneously breathing patients when compared with mechanically ventilated patients. These results validate the outcomes of study carried by Abdelwahab et al., who concluded that ultrasonic evaluation of IVC is more helpful in patients who were breathing spontaneously. ${ }^{7}$

Oguzhan et al. also stated the usefulness of bedside ultrasound evaluation of IVC in patients admitted in Emergency Department, with spontaneous respiration. ${ }^{8}$ However, they could not found any statistically significant association between IVC measurements and CVP in mechanically ventilated patients. ${ }^{8}$ Karacabey et al. evaluated the strong correlation between IVC diameters and CVP in intubated patients, as assessed by ultrasound. ${ }^{9}$ 
Table II: CVP values with corresponding IVC expiratory diameter and collapsibility index.

\begin{tabular}{|c|c|c|c|c|}
\hline \multirow{3}{*}{$\begin{array}{l}\text { CVP } \\
\mathrm{Cm} \mathrm{H} \mathrm{H}_{2} \mathrm{O}\end{array}$} & \multicolumn{2}{|c|}{ IVC maximum diameter (mm) } & \multicolumn{2}{|c|}{ Collapsibility index (\%) } \\
\hline & \multicolumn{2}{|c|}{ Mean (SD) } & \multicolumn{2}{|c|}{ Mean (SD) } \\
\hline & Mechanical ventilation & Spontaneous breathing & Mechanical ventilation & Spontaneous breathing \\
\hline$<8$ & $16( \pm 1.94)$ & $15.0( \pm 2.07)$ & $67.6( \pm 16.3)$ & $59.8( \pm 6.4)$ \\
\hline $8-12$ & $18.3( \pm 2.98)$ & $16.4( \pm 2.1)$ & $58.1( \pm 18.4)$ & $46.9( \pm 9.4)$ \\
\hline$>12$ & $24.5( \pm 4.32)$ & $26.3( \pm 7.39)$ & $31.2( \pm 7.2)$ & $28.5( \pm 10.5)$ \\
\hline
\end{tabular}

Table III: Corelation of inferior vena cava maximum diameter and collapsibility index with central venous pressure.

\begin{tabular}{|l|c|c|}
\hline & $\begin{array}{c}\text { Mechanical } \\
\text { ventilation } \\
\text { ( } \mathbf{n = 6 7 )}\end{array}$ & $\begin{array}{c}\text { Spontaneous } \\
\text { breathing } \\
\text { ( } \mathbf{n = 5 9 )}\end{array}$ \\
\hline $\begin{array}{l}\text { Inferior vena cava } \\
\text { maximum diameter }\end{array}$ & $\mathrm{R}=0.779$, & $\mathrm{R}=0.850$, \\
\hline Collapsibility index & $\mathrm{R}=-0.725$, & $\mathrm{p}=<0.001$ \\
\hline $\mathrm{p}=<0.001$ & $\mathrm{R}=-0.899$, \\
$\mathrm{p}=<0.001$
\end{tabular}

Wiryana et al. determined a strong correlation between IVC-Cl and CVP, which indicate that a rise in CVP results in reduction in IVC collapsibility index. ${ }^{10}$ Ultrasonic measurement is believed to be safe in both emergency and critical care units with frequent evaluation of clinical analyses. ${ }^{10}$ Babaie et al. carried a survey on paediatric population to find the relationship of ultrasonic evaluation of IVC (collapsibility index $(\mathrm{Cl})$ and IVC/aorta (AO) ratio) and CVP. ${ }^{11}$ They observed a convincing positive linear correlation between IVC/AO and CVP, which was statistically significant and determined that IVC/AO indices and IVC-Cl can offer a valuable guide for intravascular fluid status evaluation for critically ill paediatric patients. ${ }^{11}$

Similarly, the study carried out by Stawicki et al. determined the behaviour of CVP across relevant IVC-CI ranges and estimated the influence of positive end-expiratory pressure (PEEP) on the CVP and IVC-CI relationship. ${ }^{12}$ They observed an inverse relationship among CVP and IVC-CI and noticed that every $\mathrm{mm} \mathrm{Hg}$ of CVP corresponds to mean difference of $3.3 \%$ in IVC collapsibility. Their statistics supported the belief that small collapsibility is constant with hypervolemia or normovolemia and elevated collapsibility is consistent with intravascular fluid diminution. ${ }^{12}$ Further, they also observed the effect of PEEP on IVC-CI and noticed that as the patients without PEEP (i.e. on spontaneous breathing) had more collapsibility when matched with the patients with PEEP (i.e. on mechanical ventilation); and thus perceived greater mean collapsibility index in nonintubated patients as compared to intubated patients. ${ }^{12}$ This observation also matches present study results.

Shalaby et al. found noteworthy correlation amongst CVP and maximum diameter of IVC and IVC-CI. ${ }^{13}$ They also determined that this relationship is most favourable in calculating CVP $<10 \mathrm{~cm} \mathrm{H}_{2} \mathrm{O}$; and further recommended to use ultrasonography of IVC as a practicable guide for fluid responsiveness in patients of $\mathrm{ICU} .{ }^{13}$ Garg and his colleagues evaluated the effectiveness of ultrasonographically calculated IVC-CI in association with central venous pressure in patients with septic shock, who required ventilatory support. ${ }^{14}$ They concluded that for fluid resuscitation, CVP and IVC Cl are negatively correlated; and ultrasound may be benefitted as an effective tool to determine fluid resuscitation. ${ }^{14}$

Kacar et al. evaluated the relationship of CVP and IVC-Cl in intubated ICU patients in CPAP mode with spontaneous breathing, and established that both parameters can be used interchangably for estimation of intravascular fluid condition. ${ }^{15}$ These results are also in agreement with Azzam, who studied the relationship of IVC diameters and CVP in patients with acute circulatory failure and proved statistically that IVC-Cl is sensitive and safe alternative to CVP for assessing fluid status in these acutely ill patients. ${ }^{16}$

A suggestive relation has been noted between CVP and IVC in various studies. Mohammed and colleagues studied CVP and IVC diameters in emergency area in patients with shock and also determined good correlation among these factors. ${ }^{17}$ Rao et al. enrolled sick neonates who were requiring rigorous hemodynamic monitoring; and revealed significant negative linear correlation between IVC-Cl and CVP. ${ }^{18}$ They also endorsed the use of ultrasound as a guide for vasopressor and fluid therapy in managing shock in neonates. ${ }^{18}$ Killu et al. also evaluted that one can reliably access IVC volume status by ultrasound measurements and it is statistically corelated with standard measures. ${ }^{19}$

Millington analysed that it is possible that IVC measurements will finally be perceived as a current era CVP; quickly accepted not because it is precise but because it is simple and non-invasive. ${ }^{20}$

There are few limitations to the generalisation of results of this study. First, the patients were enrolled without regard to type and severity of disease. Secondly, CVP was measured using U-tube manometer instead of transducer, which is more accurate but not easily available. It is an outdated way of measuring CVP, but was used due to limited resources. Lastly, both CVP and ultrasound measurement are subjective, and may be influenced by personal opinion, and may also vary from patient to patient. Ultrasound results may be affected by image quality or anatomical difficulties like fat or gas in abdomen or wound dressing. 


\section{CONCLUSION}

Evaluation of IVC diameter and its collapsibility index is an easy and non-invasive method to evaluate intravascular volume status of critically ill patients; and its use is more helpful in patients who are spontaneously breathing than those who are mechanically ventilated.

\section{ETHICAL APPROVAL:}

This cross-sectional study was carried out in Surgical ICU, Lahore General Hospital after taking ethical approval from Research Review Committee of the Institution (Research No.00-172-20).

\section{PATIENTS' CONSENT:}

Written informed consents were taken from patients, and were notified regarding the research study.

\section{CONFLICT OF INTEREST:}

The author declared no conflict of interest.

\section{AUTHOR'S CONTRIBUTION:}

AAD: Concept, data collection and analyses, manuscript writing, bibliography and review.

\section{REFERENCES}

1. Sarıtaş A, Zincircioğlu Ç, Uzun Sarıtaş $P$, Uzun U, Köse I, Şenoğlu N. Comparison of inferior vena cava collapsibility, distensibility and delta indices at different positive pressure supports and prediction values of indices for intravascular volume status. Turk J Med Sci 2019; 49(4):1170-8. doi: 10.3906/sag-1810-52.

2. Ciozda W, Kedan I, Kehl DW, Zimmer R, Khandwalla R, Kimchi A. The efficacy of sonographic measurement of inferior vena cava diameter as an estimate of central venous pressure. Cardiovasc Ultrasound 2016; 14(1):33. doi:10. 1186/s12947-016-0076-1.

3. Ilyas A, Ishtiaq W, Assad S, Ghazanfar H, Mansoor S, Haris $\mathrm{M}$, et al. Correlation of IVC Diameter and Collapsibility Index With Central Venous Pressure in the Assessment of Intravascular Volume in Critically III Patients. Cureus 2017; 9(2): e1025. doi: 10.7759/cureus.1025.

4. Kent A, Patil P, Davila V, Bailey JK, Jones C, Evans DC, et al. Sonographic evaluation of intravascular volume status: Can internal jugular or femoral vein collapsibility be used in the absence of IVC visualisation? Ann Thorac Med 2015; 10(1):44-9.doi: 10.4103/1817-1737.146872.

5. Hafez AA. Measurement of Inferior vena cava collapsibility index and its correlation to central venous pressure in adult critically III patients a prospective observational study. Glob J Anes \& Pain Med 2020; 3(4): 310-8. doi: 10.32474/GJAPM.2020.03.000170.

6. Pourmand A, Pyle M, Yamane D, Sumon K, Frasure SE. The utility of point-of-care ultrasound in the assessment of volume status in acute and critically ill patients. World J Emerg Med 2019; 10(4): 232-8. doi:10.5847/wjem.j. 1920-8642.2019.04.007.

7. Abdelwahab HW, El-Wahab RA. Correlation of bedside ultra- sonographic measurement of ivc diameter and caval index with CVP in spontaneously breathing and mechanically ventilated patients. J Emerg Med Critical Care 2017; 3(1):2.

8. Citilcioglu S, Sebe A, Ay MO, Icme F, Avci A, Gulen M, et al. The relationship between inferior vena cava diameter measured by bedside ultrasonography and central venous pressure value. Pak J Med Sci 2014; 30(2):310-5. doi: 10.12669/pjms.302.4375.

9. Karacabey S, Sanri E, Guneysel O. A non-invasive method for assessment of intravascular fluid status: Inferior vena cava diameters and collapsibility index. Pak J Med Sci 2016; 32(4):836-40. doi: http://dx.doi.org/10.12669/pjms. 324.10290

10. Wiryana M, Sinardja IK, Aryabiantara IW, Senapathi TG, Widnyana IMG, Aribawa IGNM, et al. Central venous pressure correlates with inferior vena cava collapsibility index in patients treated in intensive care unit. Bali J Anesthesiol 2017; 1(1):7-9. Doi:10.15562/bjoa.v1i1.2.

11. Babaie S, Behzad A, Mohammadpour M, Reisi M. A comparison between the bedside sonographic measurements of the inferior vena cava indices and the central venous pressure while assessing the decreased intravascular volume in children. Adv Biomed Res 2018; 7:97.

12. Stawicki SP, Adkins EJ, Eiferman DS. Prospective evaluation of intravascular volume status in critically ill patients: Does inferior vena cava collapsibility correlate with central venous pressure? J Trauma Acute Care Surg 2014; 76(4):956-63; discussion 963-4. DOI: 10.1097/ta.000000 0000000152.

13. Shalaby M, Roshdy H, Elmahdy W, El Mezayen A. Correlation between central venous pressure and the diameter of inferior vena cava by using ultrasonography for the assessment of the fluid status in intensive care unit patients. The Egyptian Journal of Hospital Medicine 2018; 72(10): 5375-84. doi: 10.21608/ejhm.2018.11283.

14. Garg M, Sen J, Goyal S, Chaudhry D. Comparative evaluation of central venous pressure and sonographic inferior vena cava variability in assessing fluid responsiveness in septic shock. Indian J Crit Care Med 2016; 20:708-13.

15. Kivilcim Kacar C, Uzundere O, Bicak M, Kandemir D, Kaya $\mathrm{S}$, Yektas A. Evaluation of the relationship of Delta-CO2 with IVC-CI,IJV-CI, and CVP values in intubated critically ill patients with spontaneous breathing, and who were applied invasive mechanical ventilation in CPAP mode. Ann Med Res 2020; 27(12):3222-7. DOI: 10.5455/annalsmedres.2020. 03.270.

16. Azzam R. The role of ultrasound guided IVC-CI versus central venous pressure in assessment of critically ill patients Med J Cairo Univ 2021; 89(2):597-602 www. medicaljournalofcairouniversity.net.

17. Mohammed MA, Hagag MG, Mousa WA, Toulan MT. Correlation of inferior vena cava diameter and collapsibility index with central venous pressure in shocked patients. Menoufia Med J 2020; 33:1304-8.

18. Kumar Rao Y, Arora S, Midha T, Rao N. Correlation of ultrasonographic measurement of inferior vena cava collapsibility index with central venous pressure in diagnosis and management of neonatal shock. Iranian Journal of Neona- 
tology IJN 2020; 11(3):86-91. doi: 10.22038/ijn.2020.44748. 1743.

19. Killu K, Coba V, Blyden D, Munie S, Dereczyk D, Kandagatla $P$, et al. Sonographic assessment of intravascular fluid estimate (SAFE) score by using bedside ultrasound in the inten- sive care unit. Crit Care Res Pract 2020; 9719751. doi: 10.1155/2020/9719751.

20. Millington SJ. Ultrasound assessment of the inferior vena cava for fluid responsiveness: Easy, fun, but unlikely to be helpful. Can J Anesth 2019; 66:633-8. doi.org/10.1007 s12630-019-01357-0. 